\title{
Role of the wetting layer in the enhanced responsivity of InAs/GaAsSb quantum dot infrared photodetectors
}

\author{
Álvaro Guzmán, Kenii Yamamoto, J. M. Ulloa, J. M. Llorens, and Adrian Hierro
}

\begin{abstract}
InAs/GaAs ${ }_{1-x} \mathrm{Sb}_{\mathrm{x}}$ Quantum Dot (QD) infrared photodetectors are analyzed by photocurrent spectroscopy. We observe that the integrated responsivity of the devices is improved with the increasing Sb mole fraction in the capping layer, up to 4.2 times for $x=17 \%$. Since the QD layers are not vertically aligned, the vertical transport of the carriers photogenerated within the QDs takes place mainly through the bulk material and the wetting layer of the additional QD regions. The lower thickness of the wetting layer for high $\mathrm{Sb}$ contents results in a reduced capture probability of the photocarriers, thus increasing the photoconductive gain and hence, the responsivity of the device. The growth of not vertically aligned consecutive QD layers with a thinner wetting layer opens a possibility to improve the performance of quantum dot infrared photodetectors. (C) 2015
\end{abstract}

In the last two decades, quantum well (QW) and quantum dot (QD) infrared photodetectors have acquired high interest due to their important advantages as active elements in focal plane arrays. ${ }^{1,2}$ These advantages include access to mature GaAs technology, high detection speed, excellent reproducibility, homogeneity, or accurate control of the band structure and hence spectral response. Furthermore, QD detectors can in theory be operated at room temperature and they are sensitive to normally incident light. ${ }^{3}$ Nevertheless, these potential advantages of the QD detectors are controversial and they are still under discussion. ${ }^{4}$

In 2012, Huang et al. ${ }^{5}$ reported an improved photoresponse in QD Infrared Photodetector (QDIP) structures with 10 InAs QD layers in the active region. The dots were capped with GaAsSb barriers, and they varied the Sb mole fraction up to $20 \%$. They attribute this behavior to the presence of the GaAsSb strain reducing layer. However, a direct relationship between this improvement in the photoresponse and the physical processes taking place within the material has not been found in the literature.

In this paper, we propose a suitable model to explain this enhancement in the photocurrent based on the characteristics of the QD layers and the photoconductive gain of the whole structure. In general, the responsivity of photonic detectors is proportional to the quantum efficiency and the photoconductive gain $(g){ }^{6}$ A common expression for $g$ based on a transport model is

$$
g=L / l,
$$

where $L$ is the photoelectron mean free path and $l$ is the length of the active region. ${ }^{7}$ Moreover, for a QW detector, the gain can be obtained from (1) in terms of a well capture probability $\left(p_{c}\right)$ with the QW treated as a single trap. ${ }^{8}$ In this case, if $p_{c}$ is small (i.e., $p_{c} \ll 1$ ), the value of $g$ depends on $p_{\mathrm{c}}$ and the number of wells $(\mathrm{N})$ as

$$
g=\frac{1-p_{c}}{p_{c} N} .
$$

Thus, reducing the capture probability (i.e., increasing the electron mean free path $L$ ), it is possible to increase the photoconductive gain, and consequently, the responsivity of the device (see Eq. (3) later in this text). In this work, we propose a model which involves not only the QD, but also the wetting layers (WLs) of the different active zones. If the stacked QDs are not vertically aligned, the photoexcited electrons generated in one layer are swept to the contacts mainly through the GaAs barriers and the WLs of the additional periods. Thus, the value of $L$ is strongly affected by the thickness and composition of such WLs.

We analyze the photoresponse of 3 QDIP samples with 20 InAs QD layers capped with $\mathrm{GaAs}_{1-\mathrm{x}} \mathrm{Sb}_{\mathrm{x}}$. The $\mathrm{n}-\mathrm{i}-\mathrm{n}$ structured QDIPs were grown on Si-doped $n^{+}(001)$ GaAs substrates using solid-source molecular beam epitaxy. The schematic cross-sectional image of the QDIPs is shown in Fig. 1. The active region consists of 20 periods. Each period contains a $50 \mathrm{~nm}$ GaAs barrier, 2.5 monolayers (ML) InAs QDs, and $20 \mathrm{ML}$ of GaAsSb capping layer. A $\delta$-doping GaAs layer with a carrier concentration of $1.6 \times 10^{17} \mathrm{~cm}^{-3}$ and a thickness of $2.5 \mathrm{~nm}$, nominally corresponding to 1 electron per QD, was inserted $2 \mathrm{~nm}$ below each QD layer. The Sb mole fraction in the GaAsSb capping layer was nominally $0 \%, 10 \%$, and $17 \%$, (samples $\mathrm{A}, \mathrm{B}$, and $\mathrm{C}$, respectively). The typical InAs QD density is $4.0 \times 10^{10} \mathrm{~cm}^{-2}$ (also shown in Fig. 1), and the average height and diameter of the QDs measured by atomic force microscopy (AFM) are $7 \mathrm{~nm}$ and $22 \mathrm{~nm}$, respectively. The whole active region was sandwiched between 300 -nm-thick $\mathrm{n}^{+}$-type GaAs top and bottom contact layers doped with $\mathrm{Si}$ to $2 \times 10^{18} \mathrm{~cm}^{-3}$. The samples were processed into $200 \mu \mathrm{m}$-diameter photodetectors using 


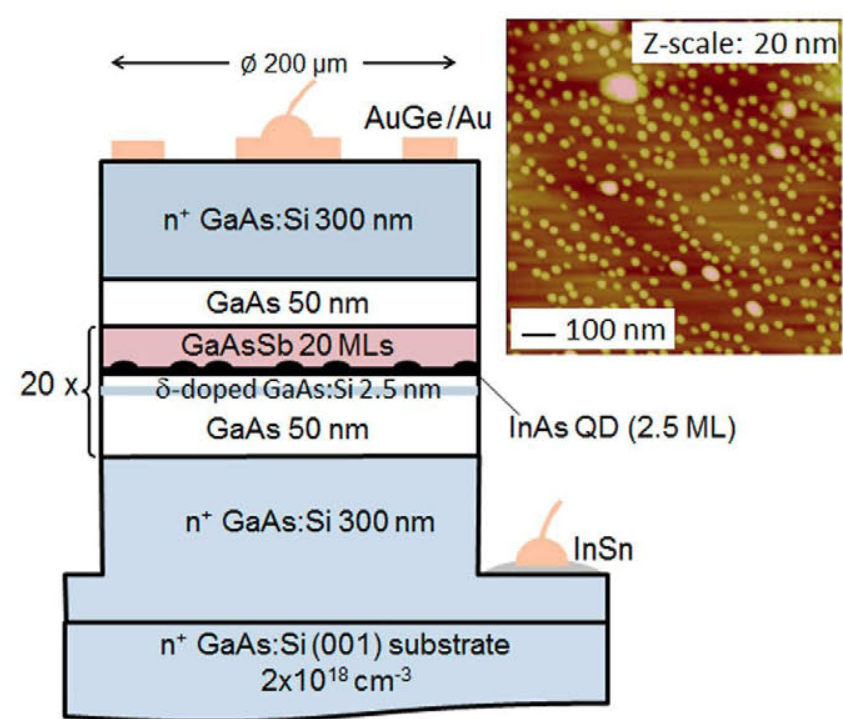

FIG. 1. Structure of the QDIP samples with 20 InAs QD layers capped with GaAsSb. The AFM image of a surface QD layer grown for test is also shown.

standard photolithography and wet chemical mesa etching. A ring-shaped metallization using $\mathrm{AuGe}-\mathrm{Au}$ was deposited on top of the mesas and then alloyed under forming gas at $350^{\circ} \mathrm{C}$ to obtain ohmic contacts. The bottom contact was chosen as the ground in all measurements. Standard tapping mode AFM measurements with Sb-doped Si tips were used to characterize the surface QDs. Photoresponse was measured by means of a Nicolet 760 Fourier Transform Infrared Spectrometer (FTIR) using a glowbar as the light source and a $\mathrm{KBr}$ beam splitter.

A key aspect in devices based on $\mathrm{Sb}$ is the interplay between In and $\mathrm{Sb}$, either in the capping layer ${ }^{9}$ or inside of the quantum dot. ${ }^{10}$ The cross-sectional scanning tunneling microscopy (X-STM) is a critical technique for determining the distribution of the chemical species. Measurements of QD layers grown under the same conditions than those studied here have shown that the GaAsSb capping layer modifies the resulting WL. The details about the measurements are published by the authors in Ref. 11. Under high voltage conditions, the STM contrast reveals mainly the topography of the outward relaxation of the cleaved surface due to the compressive strain from the QDs, the WL and the capping layer. The relaxation profile can be simulated by a finite element calculation which solves the three-dimensional problem. ${ }^{11}$ In this case, both the WL and the GaAsSb capping layer were included in the calculation, and the In and Sb segregations were also considered. The deduced WL In content profile for the samples with $0 \%, 10 \%$ and $17 \% \mathrm{Sb}$ in the capping layer is shown in Fig. 2. The WL thickness can be obtained by integrating the In content profile and further dividing this number by the perpendicular lattice parameter of a fully strained InAs layer (inset of Fig. 2). These results show that the thickness of the WL is significantly affected by the GaAsSb strain reducing layer. During the decomposition processes taking place during capping, the In which leaves the QDs moves back to the WL. Nevertheless, the GaAsSb capping layer reduces these erosion processes resulting in taller QDs and a thinner WL. Moreover, the attenuation of

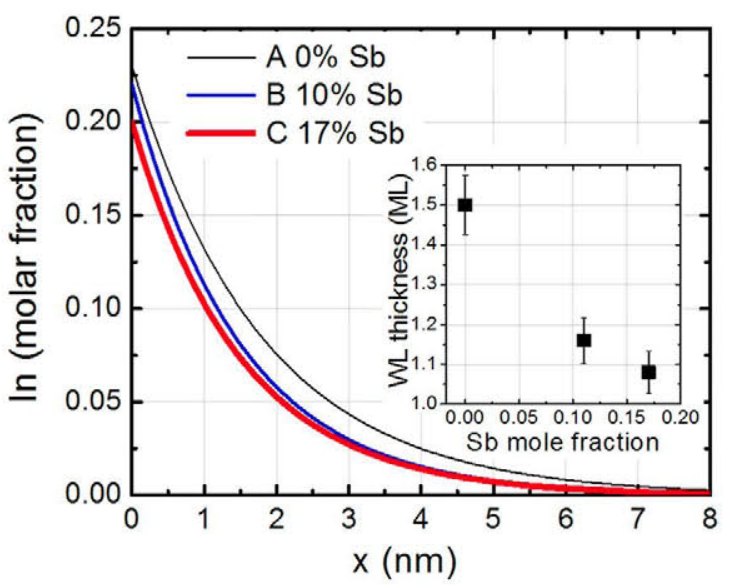

FIG. 2. Estimation of the WL thickness based on X-STM measurements of the outward relaxation of the cleaved surface. The WL thickness in ML (shown in the inset) has been obtained integrating the In content profile and dividing by the perpendicular lattice parameter of a fully strained InAs layer.

the mass transport process is proportional to the $\mathrm{Sb}$ content in the capping layer, being completely suppressed for $\mathrm{Sb}$ contents above $14 \%{ }^{11}$ Therefore, the WL thickness decreases gradually with the $\mathrm{Sb}$ content up to $14 \%$ and then remains constant. ${ }^{11} \mathrm{~A}$ WL thickness of $1.48 \mathrm{ML}$ for sample A, 1.16 ML for sample B, and 1.06 ML for sample $\mathrm{C}$ has been estimated.

The thickness of the WL is a critical factor to explain the enhancement of the intersubband photocurrent with the $\mathrm{Sb}$ content. If we analyze the carrier transport through the device we conclude that, while the photocarriers are generated within the QD, the vertical transport of such carriers takes place mostly through the bulk material and the WL-capping layer of the additional QD. Since the GaAs separating barrier is $50 \mathrm{~nm}$-thick, the QD contained in the stacked regions are not vertically aligned, therefore, it is very unlikely for the carriers to cross more than one QD in their path to the contacts. Indeed, taking into account the QD density of and size measured by AFM, the total QD effective capture cross-section is only $15 \%$ of that of the WL-capping layer. Since $\mathrm{Sb}$ is known to affect mainly the valence band of GaAs, ${ }^{12}$ the GaAsSb capping layer will not create a potential well for electrons and carrier re-trapping processes will be linked to the WL conduction band itself.

In Fig. 3, we display the potential profile along the growth direction of one of the QDs of sample A. (The black arrow in the inset indicates the equivalent region along the semiconductor sample.) A realistic QD size, shape, and composition derived from X-STM measurements are considered in the calculations. ${ }^{11}$ The QD model takes into account the impact on the electronic structure of the strain distribution resulting of the different lattice constants of the materials involved in the formation of the nanostructure and its associated linear piezoelectric field and uses an $8 \times 8 \mathbf{k} \cdot \mathbf{p}$ method. ${ }^{13}$ This model has been used in the past in the study of the electronic structure of InAs QDs capped with $\mathrm{GaAsSb}^{14}$ and containing $\mathrm{Sb}$ inside. ${ }^{15}$

Furthermore, also in Fig. 3, we show the electron ground state (GS) and the first bound state (1ES) in the QD. The simulation reveals that actually there are more bound states 


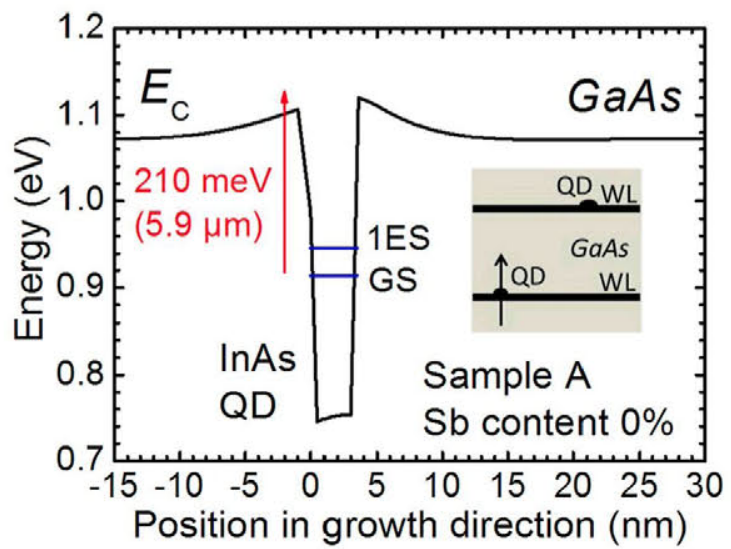

FIG. 3. Potential profile of the conduction band of a QD in sample A along the growth direction (one period of the active region). The inset displays the structure of one period where the simulated region is indicated by the black arrow.

in the QD, however, the difference in energies between two consecutive levels is less than $30 \mathrm{meV}$ in any case (which corresponds to intersubband transitions above $\lambda=41 \mu \mathrm{m}$ ). We focus our analysis only in the atmospheric transmission window $(3-5 \mu \mathrm{m})$. This wavelength range can be reached taking advantage of the transition from the GS to the continuum in the QD. In our case, for sample A, this corresponds to a difference in energies of $210 \mathrm{meV}$. According to this value, we expect an absorption peak at $5.9 \mu \mathrm{m}$ for the QD of sample A. Similar calculations have been performed for samples B and C, although they have been omitted here for simplicity. For those samples, the calculated absorption peaks are: $5.2 \mu \mathrm{m}$ in sample B and $5.0 \mu \mathrm{m}$ in sample C.

The low temperature $(12 \mathrm{~K})$ responsivity of the samples is shown in Fig. 4. The spectra have been taken by FTIR in normal incidence conditions. The peaks corresponding to the QD intersubband transitions at $\sim 5 \mu \mathrm{m}$ are clearly visible. The resulting absorption peaks match reasonably well with the calculated energies for the transition from the GS to the continuum. Besides, there is an improvement in the integrated intensity of a factor of 4.2 between samples A and C. As it was aforementioned, we attribute this improvement in

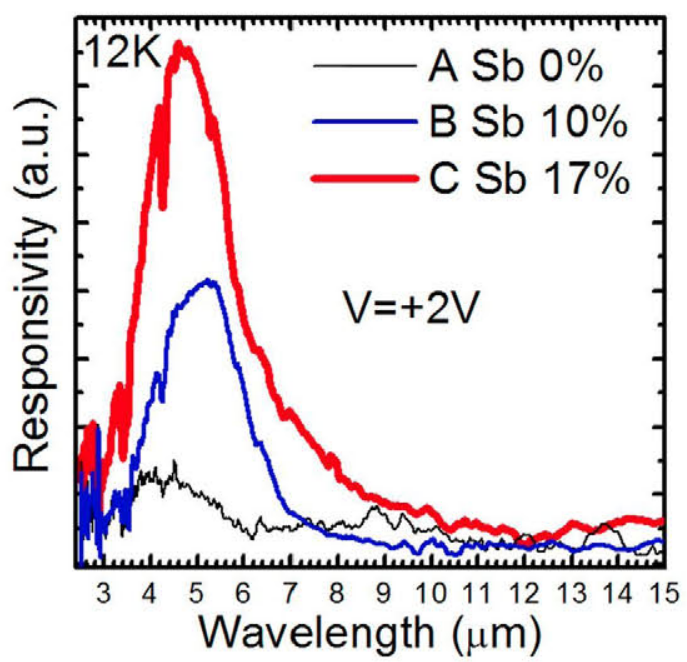

FIG. 4. Low temperature ( $12 \mathrm{~K})$ normal incidence responsivity of QDIPs A, $\mathrm{B}$, and $\mathrm{C}$ as a function of wavelength. The integrated value increases with the $\mathrm{Sb}$ content due to the increment in the photoconductive gain $(g)$. the responsivity to the thinner WLs of the samples with higher Sb content, since the photoelectrons are swept towards the contacts through those WLs. The responsivity of a photonic detector depends on the wavelength $(\lambda)$, the quantum efficiency $(\eta)$, and the photoconductive gain $(g)$ as ${ }^{16}$

$$
R=\frac{q}{h c} \lambda \eta g
$$

Since the conduction band is barely affected by the presence of $\mathrm{Sb}$ and the position in energy of the ground state does not change significantly in the samples, the variation in the quantum efficiency $(\eta)$ is expected to be very small. Therefore, assuming that the quantum efficiency is approximately equal in the three samples and that the slight variations in the peak wavelength are negligible, we find that the enhancement of the integrated responsivity must be associated with the photoconductive gain. The capture probability $\left(p_{\mathrm{c}}\right)$ can be expressed as

$$
p_{c}=1-\exp \left(-l_{w} / L_{c}\right),
$$

where $l_{w}$ is the QW width and $L_{C}$ is a decay constant. ${ }^{17}$ According to this expression, $p_{\mathrm{c}}$ increases with the well width, supporting our model: the thinner $\mathrm{WL}$ gives rise to a higher gain as deduced from Eq. (2). In Table I, we show the ratio of the integrated responsivity of samples $B$ and $C$ with respect to that of $\mathrm{A}$, together with the ratio of the photoconductive gain $(g)$ calculated from Eqs. (2) and (4) taking $l_{w}$ as the WL thickness. The values obtained using the theoretical model are in good agreement with the experimental values, showing a raising tendency of $g$ when the Sb molar fraction is increased. This indicates that the WL thickness is the critical factor that explains the enhanced responsivity of these devices.

In conclusion, we find a raising tendency of the integrated responsivity of InAs/ $\mathrm{GaAs}_{1-x} \mathrm{Sb}_{\mathrm{x}}$ quantum dot infrared photodetectors with the increasing Sb mole fraction in the capping layer. This value is as high as 4.2 times for $\mathrm{x}=17 \%$ with respect to $\mathrm{x}=0 \%$. While the photogenerated carriers are created at the QD, the fact that the QD layers are not vertically aligned makes the vertical transport of these carriers to take place mainly through the bulk material and the WL-capping layer. The lower thickness of the WL for high $\mathrm{Sb}$ contents results in a reduced capture probability of the photocarriers, and hence, in an improvement of the device responsivity. Consequently, the growth of consecutive not vertically aligned QD layers with a reduced WL thickness gives rise to the possibility of developing high performance quantum dot detectors.

TABLE I. Integrated responsivity ratio of samples B and C with respect to that of A, and photoconductive gain ratio calculated from Eqs. (2) and (4).

\begin{tabular}{lccc}
\hline \hline Sample & $\begin{array}{c}\text { WL } \\
\text { thickness }\end{array}$ & $\begin{array}{c}\text { Integrated responsivity } \\
\text { ratio with respect } \\
\text { to sample A }\end{array}$ & $\begin{array}{c}\text { Photoconductive } \\
\text { gain }(g) \text { ratio calculated } \\
\text { from Eqs. (2) and (4) }\end{array}$ \\
\hline A & $1.48 \mathrm{ML}$ & 1 & 1 \\
B & $1.16 \mathrm{ML}$ & 2.5 & 3.0 \\
C & $1.06 \mathrm{ML}$ & 4.2 & 4.2 \\
\hline \hline
\end{tabular}


This work has been supported by the Comunidad de Madrid through Project No. S2013/MAE-2780 and by the Spanish Ministry of Economy and Competitiveness through Project Nos. MAT2013-47102-C2-2-R, ENE2012-37804C02-02, and AIC-B-2011-0806.

${ }^{1}$ A. Rogalski, J. Antoszewski, and L. Faraone, J. Appl. Phys. 105, 091101 (2009).

${ }^{2}$ B. F. Levine, J. Appl. Phys. 74, R1 (1993).

${ }^{3}$ H. C. Liu, M. Gao, J. McCaffrey, Z. R. Wasilewski, and S. Fafard, Appl. Phys. Lett. 78, 79 (2001).

${ }^{4}$ H. C. Liu, Opto-Electron. Rev. 11, 1 (2003).

${ }^{5}$ C.-T. Huang, Y.-C. Chen, and S.-C. Lee, Appl. Phys. Lett. 100, 043512 (2012).

${ }^{6}$ B. F. Levine, A. Zussman, S. D. Gunapala, M. T. Asom, J. M. Kuo, and W. S. Hobson, J. Appl. Phys. 72, 4429 (1992).

${ }^{7}$ G. Hasnain, B. F. Levine, S. Gunapala, and N. Chand, Appl. Phys. Lett. $\mathbf{5 7}, 608(1990)$

${ }^{8}$ H. C. Liu, Appl. Phys. Lett. 60, 1507 (1992).
${ }^{9}$ J. M. Ripalda, D. Granados, Y. González, A. M. Sánchez, S. I. Molina, and J. M. García, Appl. Phys. Lett. 87, 202108 (2005).

${ }^{10}$ A. G. Taboada, A. M. Sánchez, A. M. Beltrán, M. Bozkurt, D. AlonsoÁlvarez, B. Alén, A. Rivera, J. M. Ripalda, J. M. Llorens, J. MartínSánchez, Y. González, J. M. Ulloa, J. M. García, S. I. Molina, and P. M. Koenraad, Phys. Rev. B 82, 235316 (2010).

${ }^{11}$ J. M. Ulloa, R. Gargallo-Caballero, M. Bozkurt, M. del Moral, A. Guzmán, P. M. Koenraad, and A. Hierro, Phys. Rev. B 81, 165305 (2010).

${ }^{12}$ R. Teissier, D. Sicault, J. C. Harmand, G. Ungaro, G. Le Roux, and L. Largeau, J. Appl. Phys. 89, 5473 (2001).

${ }^{13}$ S. Birner, T. Zibold, T. Andlauer, T. Kubis, M. Sabathil, A. Trellakis, and P. Vogl, IEEE Trans. Electron Devices 54, 2137 (2007).

${ }^{14}$ J. M. Ulloa, J. M. Llorens, M. del Moral, M. Bozkurt, P. M. Koenraad, and A. Hierro, J. Appl. Phys. 112, 074311 (2012).

${ }^{15}$ A. G. Taboada, J. M. Llorens, D. Alonso-Álvarez, B. Alén, A. Rivera, Y. González, and J. M. Ripalda, Phys. Rev. B 88, 085308 (2013).

${ }^{16} \mathrm{P}$. Bhattacharya, Semiconductor Optoelectronic Devices, 2nd ed. (Prentice Hall, Upper Saddle River, NJ, 1997).

${ }^{17}$ M. A. Gadir, P. Harrison, and R. A. Soref, J. Appl. Phys. 91, 5820 (2002). 